\title{
Téoros
}

Revue de recherche en tourisme

\section{Le paysage et le jardin}

La quête de l'événement

\section{Philippe Poullaouec-Gonidec}

Volume 18, numéro 1, printemps 1999

Les jardins du tourisme

URI : https://id.erudit.org/iderudit/1072297ar

DOI : https://doi.org/10.7202/1072297ar

Aller au sommaire du numéro

Éditeur(s)

Université du Québec à Montréal

ISSN

0712-8657 (imprimé)

1923-2705 (numérique)

Découvrir la revue

Citer cet article

Poullaouec-Gonidec, P. (1999). Le paysage et le jardin : la quête de l'événement. Téoros, 18(1), 26-31. https://doi.org/10.7202/1072297ar d'utilisation que vous pouvez consulter en ligne.

https://apropos.erudit.org/fr/usagers/politique-dutilisation/ 


\section{ta...... \\ Le PAYSAGE ET LE JARDIN \\ LA QUÊTE DE L'ÉVÉNEMENT}

\section{Philippe Poullaouec-Gonidec}

La société est avide de ses paysages. De l'afirmation identitaire des lieux en passant par le développement de destinations touristiques cibles. le paysage devient l'enjeu de l'aménagement du territoire el de l'environnement du cadre de vie. Le jardin, quant à lui, sort des murs. Forme du quotidien des villes et des territoires suburbains (parcelle résidentielle) et objet d'exception (patrimoine historique), le jardin est réinvesti par le public. Il est entré dans un court laps de temps dans les univers commerciaur, industriels' et médiatiques.

Au-delà de cette réalité connue, le paysage et le jardin sont deux formes d'expression à la fois distinctes et semblables. Leur similarité et leur filiation sont multiples. Le jardin peut être perçu comme étant la miniaturisation du monde, le réceptacle d'un imaginaire, d'une sensibilité particulière ou d'une représentation de la nature. Il est défini aussi comme étant une troisieme nature ${ }^{2}$ par 1'historien John Dixon Hunt: « L'art des jardins est la forme la plus sophistiqué de l'art du paysage [... I dont il n'est qu'une partie ». C'est un art du milieu (Hunt, 1996: 16) tout comme le paysage qui, pour sa part, est lié au phénomène d' « artialisation $\%^{3}$ in visu et in situ. Ce dernier est la qualification esthétique d'un regard posé envers un territoire ou un lieu. "Chaque espace (environnement) peut devenir un paysage par le biais d'un phénomène de médiatisation qui crée une qualification esthétique * (Poullaouec-Gonidec et Jacobs, $1992: 9$ ).

Le paysage est parfois perçu comme l'extension du jardin. Certaines conceptions vont même jusqu' ta projeter le jardin à l'échelle de la planète. Le projet de « jardin planétaire \& de Gilles Clément (1997) illustre cette extension métaphorique.
Notre jardin, celui des hommes en quête de savoir n'est pas un lieu de l'épuisement des sciences, un objet observé à distance, c'est un système. sans limite de vie, sans frontiere et sans appartenance, nourri au rêve des jardiniers et sans cesse remodele par les conditions changeantes de la nature. C'est un lieu de sauvegarde des réalités tangibles et intangibles.

Le paysage et le jardin sont souvent interpellés par le social comme étant des lieux d'expériences esthétiques et, largement, d'évasions sensorielles. La préoccupation dont ils font l'objet actuellement semble tenir de la formalisation d'expressions sans cesse renouvelées. Une mise en perspective de cette réalité permet de saisir la portée des deux phénomènes en question.

\section{LE PAYSAGE, DE L'ÉTONNEMENT À L'ÉVÉNEMENT}

Le paysage porte en lui l'idée d'événement. Il suffit de prendre comme point de départ le fait que le paysage soit intimement lié à l'invention pour s'en rendre compte. Le paysage s'invente et se réinvente au gré des sensibilités et des émotions. Ses naissances sont à lá fois le fruit de délectations esthétiques, d'appropriations, et le lieu d'affirmations individuelles et collectives apparaissant notamment lors de certaines opérations de développements ou de mutations territoriaux (ex. : la déprise agricole et l'apparition de friches végétales).

De nombreux auteurs l'ont décrit: le passage d'une réalité de pays à la réalité du paysage s'est traduit par des représentations éloquentes et magnifiées d'une idée de nature. Pour reprendre les deux archétypes classiques de l'invention paysagère du XVIII" siècle en Europe, I'expérience esthétique de la montagne et de la mer est imprégnée d'un couple indissociable et à la fois opposét que forment le sublime et le beau. A propos du beau et du sublime, Michel Tournier résume de manière concise ces éléments significatifs du sentiment esthétique :

Si le beau est fini et harmonieux, le sublime est infini et dynamique. Le sublime nous place dans tan état de déséquilibre vertigineux où se mêlent étrangement le plaisir et la terreur. Le beau relève de la qualité, le sublime de la quantite. Enfin le beau invite au jeu, à la divine gratuité d'un paradis sans obligation, ni sanction, tandis que le sublime renvoie à des notions théoriques, morales et religieuses (Tournier, 1994 : 158-159).

Edmund Burke (1990), philosophe du XVIII ${ }^{r}$ siècle, traduit très bien la notion du 
sublime telle qu'elle s'exprimait lors de l'avènement des deux paysages (montagne et océan) en occident :

Tout ce qui est propre à susciter d'une manière quclconque les idees de douleur et de danger, c'est-à-dire tout ce qui est d'une certaine manière terrible, tout ce qui rraite d'objets terribles ou agit de facon analogue à la terreur, est source du sublime, c'est-à-dire capable de produire la plus forte des entions que l'esprit soit capable de ressentir:

Ces idées de douleur, précise l'auteur, nécessitent une mise à distance et certaines modifications pour qu'elles soient perçues comme des experriences délicieuses. Il ajoute que l'une des clés de cette expérience est l'étonnement; cet « étonnement (...) est l'effer du sublime à son plus haut degré; les effets inferieurs en sont l'admiration, la vénération et le respect $\$$ (Burke, 1990).

Cet attribut de l'expérience paysagère qu'est l'étonnement pourrait s'associer à l'idée du spectaculaire, * L'étonnement est l... l'apparinon de l'extraordinaire dans l'ordinaire, (Ronfard, 1998: 52). La notion de paysage apparticndrait ainsi à ce qui parle aux yeux, en impose à l'imaginaire ${ }^{5}$. En effet, il semble que vis-à-vis du paysage, nous soyons toujours en quête de sensations, d'événements annoncés ou, plus précisément, mis en scène.

L'avènement de la conception purement visuelle du paysage nord-américain dans les années 1970 est l'un des exemples les plus significatifs de la thédralité payságère. Celle-ci s'est exprimée dans le dessin des autoroutes scéniques où l'usager était un spectateur mis à distance d'un scénario bien calculé tout au long d'un trajet. En effet, les découvertes paysageres d'un réseau de transport offrent des cadrages de plans, des équilibres de masses et de textures biotiques et abiotiques, des profondeurs infinies et des aplats, le tout perçu à travers une perspective cinétique où $\alpha$ se mélangent des premiers plans, aux mouvements les plus rapides, des plans intermédiaines aux mouvements contradictoires (parfois même rotatifs) qui mènent à une relative fixite des plans les plus lointains $x$ (Lassus, $1994: 54)$.

Cet art de l'in visu est en quelque sorte un art de la transposition du genre pictural et

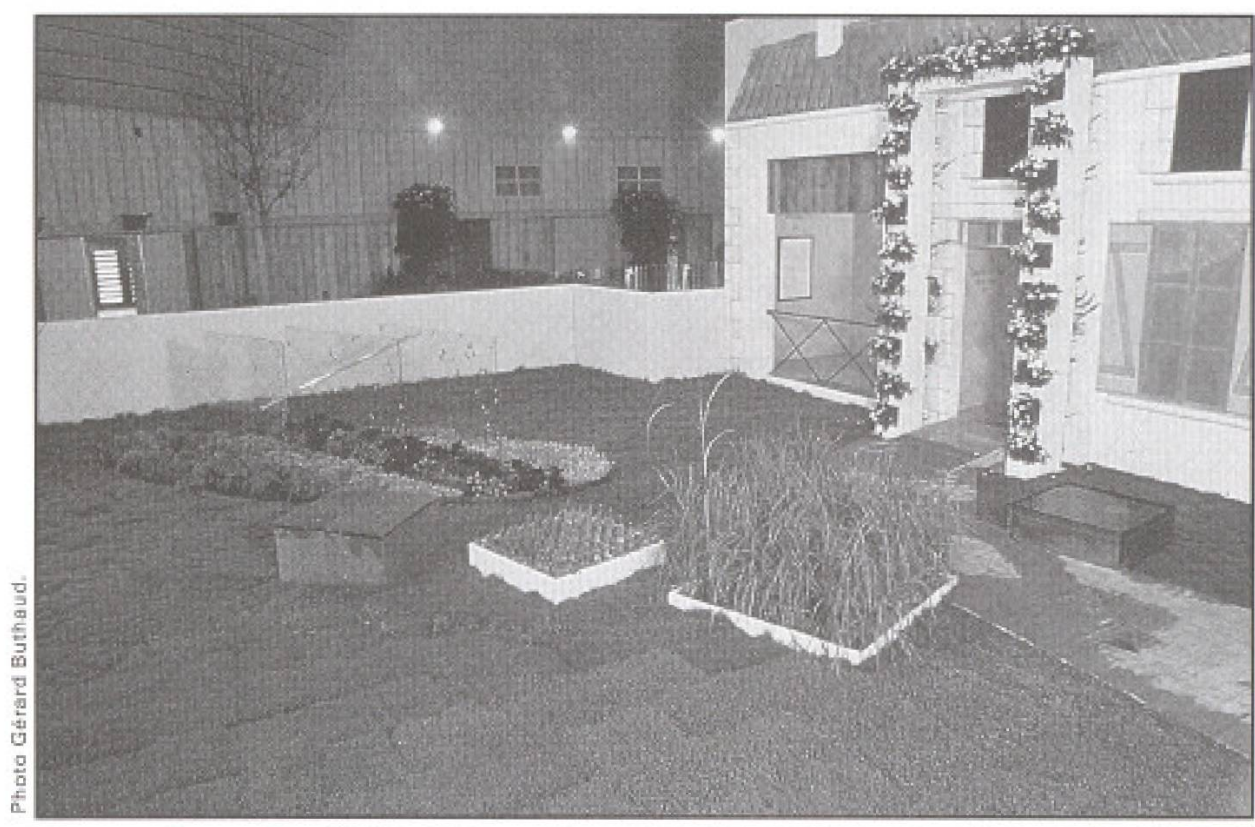

Hommage à monsieur Hulot de Franco Zagari (Jardins événementiels de Niort - 1993).

des principes de découvertes dessinés dans les parcs et les jardins anciens, Ce tout est en fait une résurgence mesurée du pittoresque. Car il s'agit bien de mesure, de calcul et de géométrie qui nous mènent bien au-delà d'une simple délectation esthétique ou même de l'idée de beauté telle que l'exprimait Emund Burke à son époque. La réflexion actuelle sur la requalification des corridors routiers et autoroutiers significatifs du Québec ${ }^{\circ}$ a toutefois un autre dessein : celui de construire les termes d'un projet de paysage imprégné de la sensibilité des lieux et coupé des modèles traditionnels de l'in visu,

De l'autoroute scénique à l'aménagement des sentiers de decouverte de la belle nature des parcs nord-américains, le paysage evénement offre des mises en scène communes où la vue est souveraine comme pour assouvir un public à la recherche de poims de vue. Cette attitude contemplative esthétisante du public semble se satisfaire d'un spectacle sans cesse répété. De plus, la mise en scène suppose l'acquisition d'aptitudes à regarder. Ainsi, le point de vue est devenu au fil du temps didactique, c'est-à-dire explicatif de ce qui peut être observé. L'ailleurs est décrit par rapport à l'ici, l'histoire des lieux se superpose au présent et les éléments de la nature deviennent l'un des tableaux centraux du paysage qui mène au développement d'une esthétique environnementale (Poullauouec-Gonidec, 1993). L'événement paysage est devenu source de délectations esthétiques multiples, superposées, surinvesties de sens; un concentré saisi dans l'instantané, le temps d'une photographie prise à la va-vite, le point de vue n'étant qu'une courte ponctuation dans le défilement d"un parcours de découvertes de plus en plus effréné. Le point de vue n'est plus l'ile des lenteurs (Baridon, 1998) inscrit dans une découverte progressive du paysage. Sommes-nous face à l'émergence de nouvelles attitudes contemplatives ?

Banalisée, la wue panoramique au détour d'un sentier récréatif est faite pour être découverte et pour être ensuite revisitếe, comme pour pouvoir en retirer tous les plaisirs afin de mieux les enraciner. Cette soif du regard sur l'étendue de pays est partagée avec la recherche d'un corps à corps avec la nature. Les ruptures de pente, les falaises accidentées, les rochers démesurés dans les bois, les profondeurs obscures de la foret ainsi que les faisceaux de lumière qui la traversent constituent les quelques attributs recherchés du spectacle par un promeneur (écotouriste) en quête de rebranchement avec la matérialité d'un lieu. Au-delà de certaines conceptions dépassées de sa vision du social, Karl Gottolb Schelle (1996), avec son essai sur l'Art de la promenade, rappelle ainsi qu'il $y$ a autant de promenades que des lieux differrents et que cette activitế suggère des dispositions d'esprit particulières en fonction des milieux (la forêt, la rivière, la montagne, les pares, etc.). Se faisant, il 


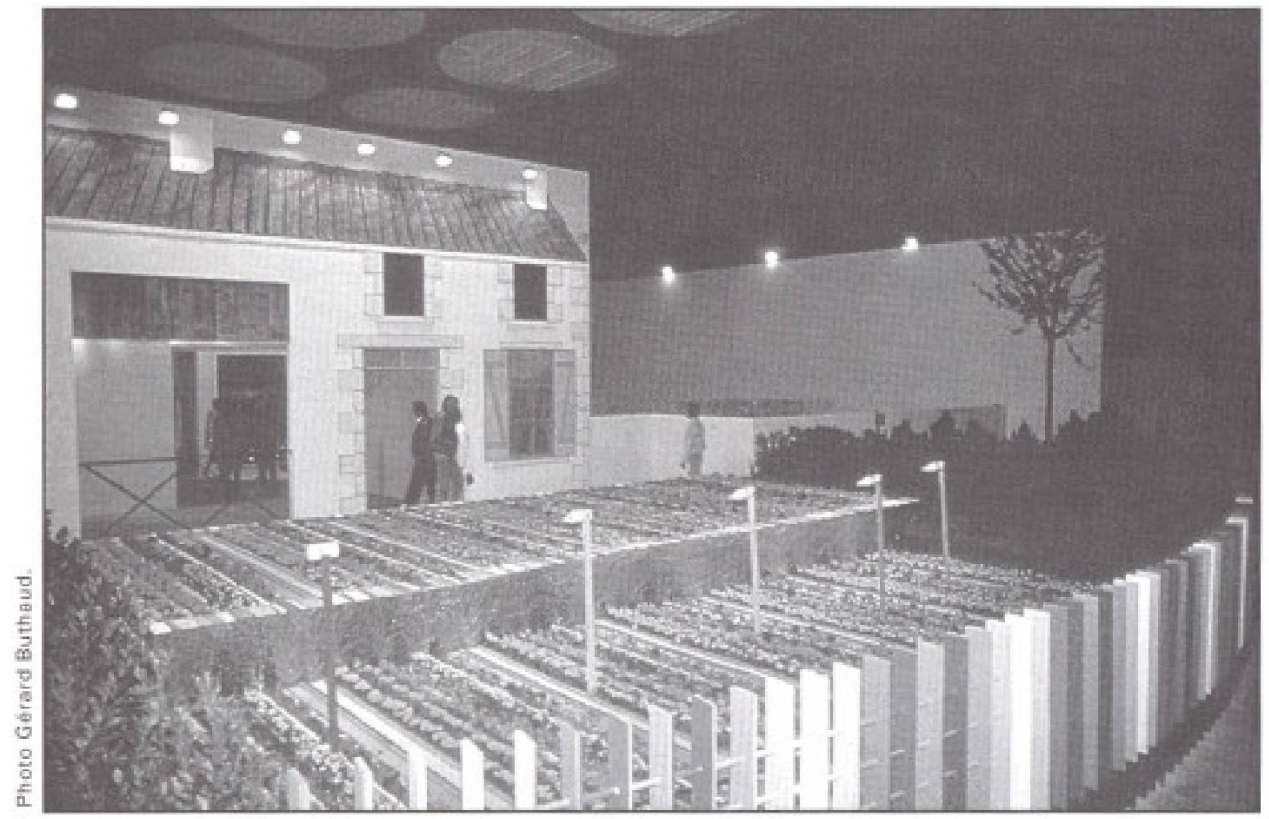

Buissons optiques de Bernard Lassus (Jardins evenementiels de Niort - 1993).

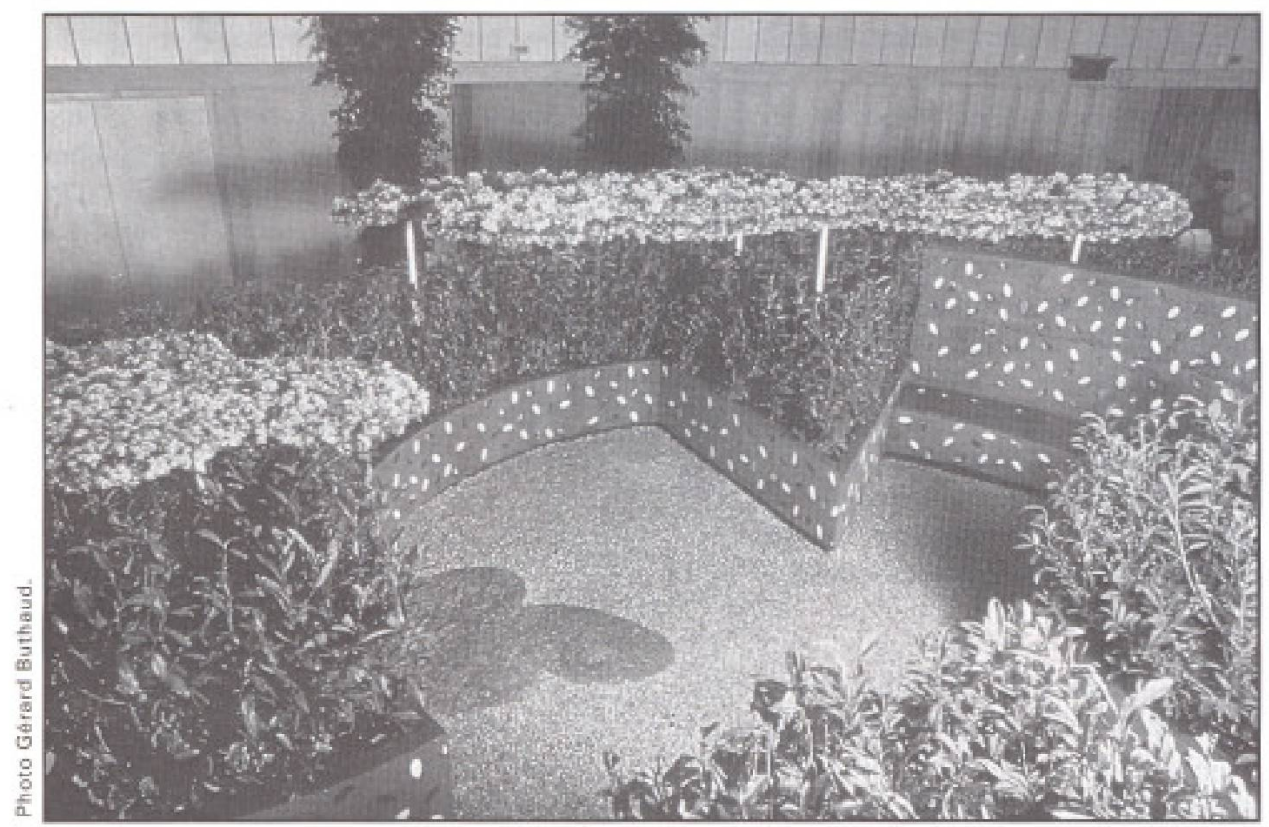

Le jardin des nuages de Philippe Poullaouec-Gonidec (Jardins événementiels de Niort-1993).

Énonce les termes d'un manuel pour atteindre les plaisirs impressionnistes de cet art :

\section{[...] les montagnes exalrent I'ima-} gination et delivrent du sol qui porte le corps, quand on se promène dans une belle vallée et que l'on se laisse aller a l'impression qu'elle degage. on se sent au contraire retenu dans le cercle de son existence et I'on savoure avec une tranquille delectation les objets qui nous entouren (Schelle, 1996: 89).
Cette relation directe, filiale et voire conbative avec la mère nature semble nécessaire pour provoquer l'émotion et le sentiment esthétique. Elle s'opère bien êvidemment par d'autres formes d'investissements. Les sociologues Bernard Kalaora et Valentin Kalaora, par leurs études sur les modèles de consommation sociale et culturelle de la forêt en France, mettent en évidence un phénomène particulier, celui d'une relation de proximité où l'individu $s^{*}$ accote au plaisir sans y gouter le tréfonds in situ. De leurs observations du vert familial, ils soulignent : \& On veille particulierement sur les enfants ; on sourit des vieilles legendes, mais on craint les mauvaises nencontres et l'on ne s'éloigne pas de la route et des voitures $\%$ (1977; 129), En outre, leur recherche sur la forêt de Fontainebleau (Kaloara. 1981), démontre de quelle manière certains visiteurs éprouvent encore à la fois une crainte, mais aussi une réelle satisfaction, celle de renforcer les liens moraux et familiaux, tout en restant en lisière. "La forêt ne devient paysage au sens precis du terme. que lorsqu'elle est vue d'en dehors . (Kalaora et Pelosge : 92).

Cette consommation indirecte du paysage appartient à l'univers de nos expériences mutuelles, tout comme habiter près de la mer et n'avoir pas nécessairement le besoin ou l'envie de $s^{+} y$ aventurer pour y être intimement lié. Son cồtoiement nous suffit, nous rassure et assouvit le désir attendu d'être simplement là, tout près, et de pouvoir à tout moment y jeter un regard.

Cette singularité, analgame de ménoires et d'évocations in visu et in sifu des lieux, démontre la complexité de l'expérience paysagère. Sa condition s'associe à la fois au quotidien spectaculaire et à l'événement d'occasions; le surinvestissement visuel et la démesure imaginaire en sont les ingrédients.

\section{LE JARDIN, ART D'EXPRESSIONS ÉVÉNEMENTIELLES}

Art de la mise en scène de nos environnements de vie de cette fin de siècle, le jardin représente l'exemple le plus dloquent de l'événement spectaculaire. Fruit d'intentions multiples à travers son histoire, le jardin restera l'expression de la quête d'un paradis perdu où l'on réinvente sans cesse des idées de natures et de cultures. En effet, l'histoire des jardins des sociétés occidentales et orientales révèle la richesse de la réflexion dans ce domaine de la création. Ainsi, il existe autant de jardins que de grandes civilisations et d'époques. Des représentations de la nature, en passant par le simple plaisir sensuel, le jardin est le miroir infini des cultures et des sociétés qui l'imaginent. Comme le souligne John Dixon Hunt :

L'art des jardins (qui est la forme la plus sophistiquée de l'ant du 
paysage est pour l'homme un mode fondamental d'expressions et d'experiences. II s'agit d'un terme moderne forgé pour désigner l'intervention par laquelle, dans un espace donne, des hommes et des femmes façonnent et créent un nouvel environnement pour eux-mêmes ou pour une société ou une culture donnée (Hunt, 1996: 16).

Le jardin, au cours des millénaires, est done devenu un « art * de savoir-faire (de penser, de projeter, de construire et d'expérimenter) qui fut, notamment au Siècle des. Lumières (et ce jusqu'au siècle dernier), colligé dans de nombreux traites' ${ }^{\top}$. Paradoxalement, notre siècle n'aura pas aussi clairement contribué au développement de cet art. Par contre, il est étonnant de constater que le XX ${ }^{e}$ siecle a produit un nombre impressionnant de jardins, c'est-à-dire mon jardin, celui du voisin, ceux du quartier. On serait même tenté d'affirmer que la production vernaculaire ( 1 'amenágement des jardins privés autour des maisons individuelles) depuis plus de trente ans constitue l'un des éléments les plus représentatifs de l'art des jardins de ce siècle ${ }^{8}$. Mis à part quelques morceaux significatifs (jardins Art déco, jardins modernistes, etc.), ces expressions de jardins n'ont toutefois généré qu'un univers de réinterprétations formelles, miroir de notre condition postmoderne. Cette profusion de jardins nous aura toutefois permis de nous interroger sur la pluralité de nos rapports à la nature et à là culture des lieux.

La forme la plus événementielle du jardin nous est livrée depuis quelques décennies au Québec" , comme partout ailleurs, par les foires horticoles et les floralies. Ces lieux sont devenus les rendez-vous commerciaux d'un savoir-faire mis en vitrine. Certaines manifestations européennes, notamment en France, ont ouvert la voic au renouvellement de l'art des jardins contemporains. Le début des années 1990 aura été marqué par ce renouveau qui s'inscrivait dans la continuité d'une décennie consacrée à la redécouverte du végétal dans des manifestations telles que celles de Courson et de Saint-Jean-deBeauregard. Deux événements illustrent cette tendance dont le premier fut la Foireexposition de Niort tenue en mai 1993. Cette manifestation internationale inscrite dans la tradition des rendez-vous horticoles invitait quatre designers de paysages urbains de différents pays ${ }^{10}$ à construire

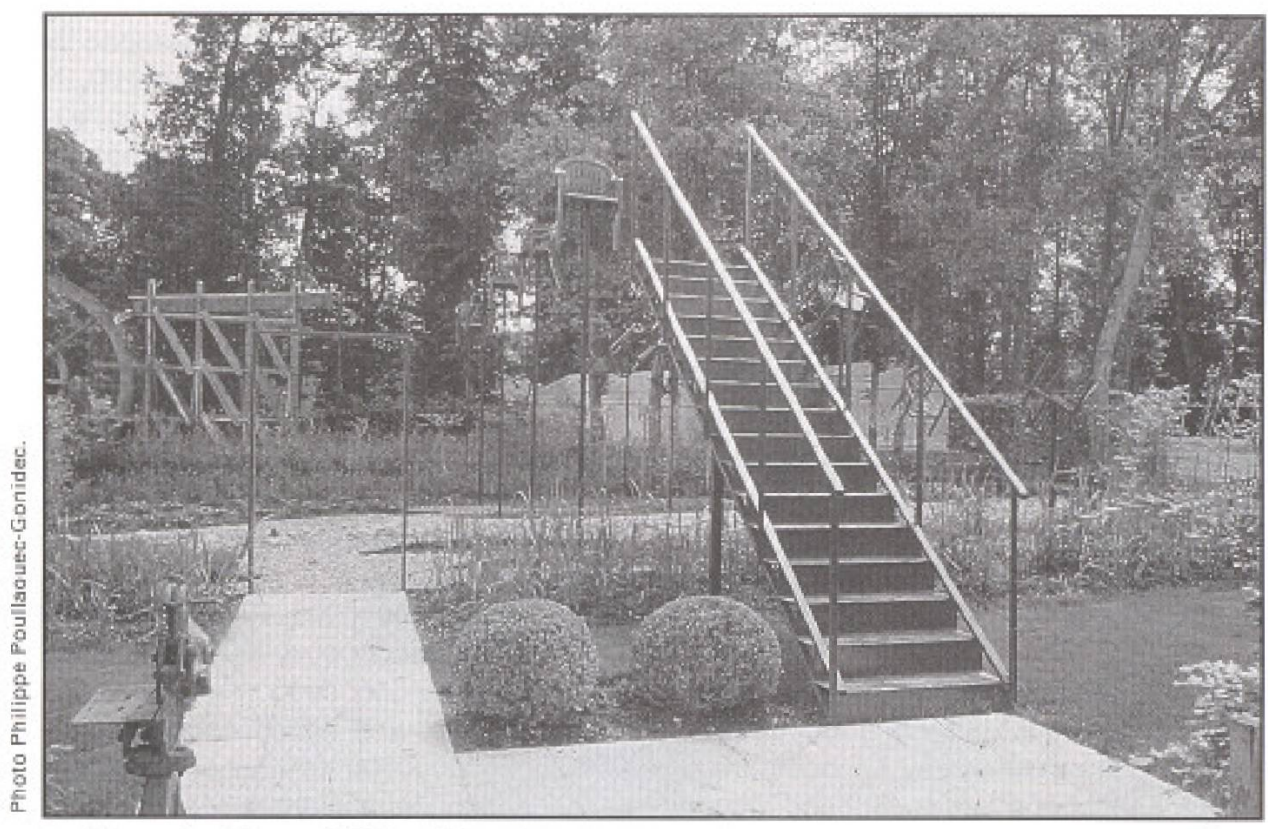

Jardin après déluge d'Édith Julien, Mélanie Mignault et Michel Langevin, étudiants de l'École d'architecture de paysage et de l'Ecole d'architecture de l'Université de Montréal. Festival international des jardins de Chaumont-sur-Loire ( $\alpha$ Ricochets w, edition 1998).

quatre jardins, quatre expressions autour d'un même thème. Le but de cette invitation à la crétion était d'étonner le visiteur par l'aspect hors du commun ei spectaculaire des projets présentés au grand public durant neuf jours. Chaque conception devait surprendre par sa créativité, son originalité, en exploitant le thème d'un jardin secret urbain : \& Le jardin retrouve son enclos dans son univers parcellaire de nos villes de pierres, ll est l'enclave dans la ville, éloigné de la vue de tous. Il est notre intimite, notre jardin secret $[\ldots]^{\prime \prime} \infty$. De cette vision quelque peu traditionnelle du jardin allaient prendre forme des concepts hétérogènes qui illustraient la volonté des créateurs de sortir des sentiers battus et de révéler certaines sensibilités perdues.

Le deuxième événement est d'une tout autre échelle. Il s'agit du Festival international des jardins de Chaumont-sur-Loire ${ }^{12}$ initié en 1992. Cette manifestation internationale se différencie des foires et des floralies dans le sens où elle est entièrement dédiée à la création de petits jardins éphémères. Elle maintient néanmoins un lien étroit avec la tradition horticole du fait que le végétal prend une place importante dans l'ensemble des créations. L'un des objectifs du festival est d'étonner le public par l'utilisation et la combinaison d'espèces végétales.

Chaque édition de ce festival rassemble des paysagistes français et étrangers. invités ou sélectionnés par concours ${ }^{13}$ autour d'un thème. Le plaisir (1992) fut le premier theme, suivi de L'imagination dans la crise (1993), Lacclimatation (1994), Jandins de curiosite (1995), La technique est-elle poétiquement correcte? (1996), One d'eau! Que d'eau! (1997) et Ricochets (1998). L'ensemble des thématiques reflète le souci d'interpeller certaines spécificités du jardin et de profiter de certaines humeurs du temps pour optimiser l'attrait de la manifestation aux yeux du grand public. Après sept années d'existence, le caractère événementiel du festival s'impose. Chaque édition du festival crée, dès son ouverture au mois de juin, un ensemble d'effets immédiatement saisissables. Les vingt-cing installations de jardins présentées annuellement au public provoquent réactions et sensibilites : le festival est un spectacle, une mise en scène où se juxtaposé une série de tableaux.

Chaque réalisation prend place dans un enclos (alvéole) en forme de feuille de tulipier. Cette assise très formelle, conçue par le paysagiste Jacques Wirtz, cerne chaque jardin éphémère. Cette division présente l'inconvénient de délimiter l'espace de création et d'encadrer les ceuvres dans une ceuvre réceptacle. Cette dernière sert-elle de faire-valoir aux ouvres ? Ou inversement les $\alpha u v r e s$ (jardins) sont-elles là pour bonifier l'aménagement (réceptacle) ? Là est toute l'ambiguité. De plus, cet encadrement formel n'empêche-t-il pas 
le visiteur de se sentir dans le lieu pour en comprendre la sensibilité intentionnelle, dans la mesure où le jardin, contrairement au paysage, souffre d'une mise à distance?

Malgré l'engouement du grand public et son succès croissant (de 60000 visiteurs À la première édition ă 147000 en 1997). le festival semble pourtant faire l'objet de réserves et de critiques de la part des paysagistes. Cet événement ne traduirait pas réellement l'idée du jardin du fait de son caractère evénementiel et ephémère. Il ne permettrait pas, entre autres, au temps de prendre racine.

Cette idéc de jardin évenementiel rejoint indéniablement les besoins d'un public en quête de renouvellement. Le jardin est le miroir expressif d'une société en mouvement. Nul ne peut ignorer qu'il appartient à l'univers quotidien du grand public. II est. pour un grand nombre d'individus, l'élément essentiel d'un chez-soi. Il exprime la qualite d'un environnement de vie (d'un art de vivre). En plus d'être soumis a l'influence des modes horticoles (coulleurs, textures, plantes annuelles et vivaces) et des regards du voisinage, il est l'expression même des gens qui y vivent.

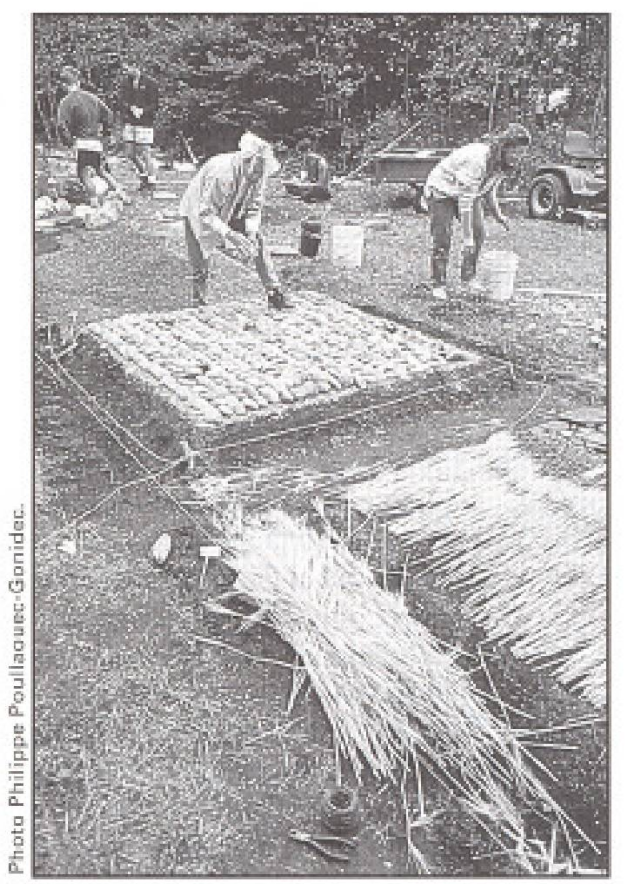

Parterres éphémères en réalisation (projet des tudiants de I'Ecole d'architecture de paysage). Concours de design des parterres. Première edition de I'École d'été de Métis (1998) organisee par l'Ecole d'architecture de paysage et la Chaire en paysage el environmenent de I'Lnivervité de Montréal.
Directement porté par le phếnomène du cocooning (ou de la tendance nest), le jardin nord-americain est avant tout l'extension naturelle (contrastée ou non) des environnements intếrieurs. De cela, il s'apparente à l'univers du décor ou de $l^{\prime}$ ornementation, voir du bel arrangement (Kant, 1993), qui justifie le déploiement d'une mise en scène sans cesse réactualisée au gré des saisons et des propriétaires.

Le jardin doit ainsi s'adapter aux besoins d'un public dont la culture est profondément marquée par le nomadisme urbain.

De déménagement en déménagement, nous perdons peut-être la mémoine des lieux. Par contre, ce trait culturel offre l'avantage particulier, celui de nous permette de porter des nouveaux regards et affects sur l'espace desquels il nous est possible de construire de nouvelles représentations paysagères (Poullaouec-Gonidec, $1993: 32$ ).

La condition ephemère du jardin, telle que promue par le Festival de Chaumont-surLoire, sinserit dans l'air du temps pré$\operatorname{sent}^{14}$. Mais, cette valorisation de l'ephemèrité n'est pas nouvelle. En effet, dans l'histoire de l'art de jardins, notamment au XVII' siecle, on trouve des pratiques similaires avec les parterres de broderie vegetale des jardins de plaisir ${ }^{15}$, concus pour l'etonnement. Les decorations florales pour le temps d'une fête prirent ainsi une importance particulière à Versailles.

Toutes les tables (dans l'un des cabinets de verdare des jardins du chateaul étaien couvertes d'une infinite de choses delicates et disposees d'une manile nouvelle; leurs pieds [...] etaient environnes de feuillages meles de feslons de fleurs [... ]. Du milieu des tables s'elewait un jet d'eau de trente pied de haut l... l' de sorte qu'en woyant tous ces buffets d'une mêne hauteur joints les uns aux autres par les branches d'arbres et les fleurs dont ils étaient revelus, il semblait que ce fat whe petite montagne du haut de laquelle sortait une fontaine (Baridon, 1998 : $774-775$ ).

A cette epoque baroque, $1^{*}$ evénement est dans la nature du jardin. Les surprises prennent une place importante, de multiples façons, par les jets d'eau et les plaisanteries

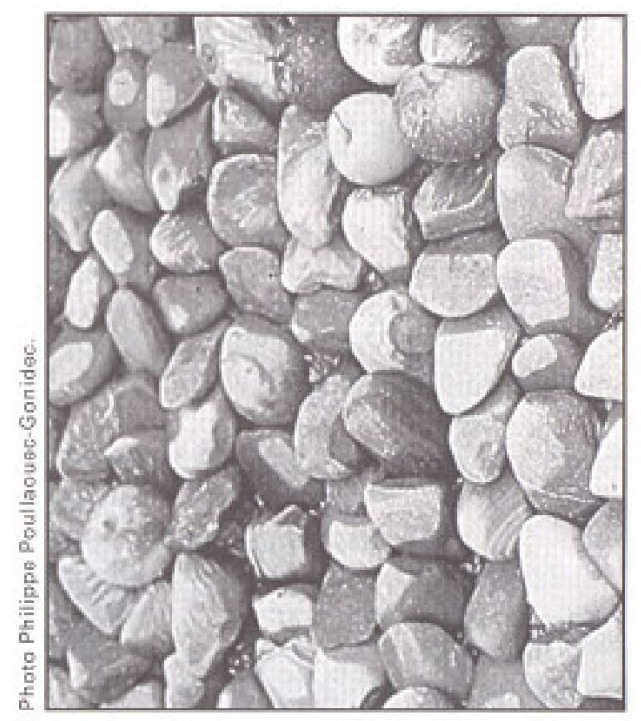

Délails du parterre (Galet de pomme, premier prix du concours de design des parterres design : Esther Bedard at Nathalie Lafrenière). École d'été de Métis (199S) organisée par l'École d'architecture de paysage et la Chaire en paysage et environuement de l'Universüte de Montréal.

hydrauliques, les automates et androïdes, les farces et les effets speciaux.

Lieu dillusion, le jardin est aussi celui de l'experience. Ainsi nul ne se prête mieux que lui à la possibilité de recréer des scènes et des phénomenes complexes destinés à enouvoir le spechateur. Les effers speciaux, mais aussi les farces, sont destines a domer du monde whe image violente, mais aussi hadique et imprévisible. [...]. Le jardin se veut ainsi la reproduction du monde dans ce qu'il a de divers, d'agréble mais aussi d'effrayant (Laroze, $1990: 219)$.

Les manifestations récentes du jardin Evenementiel sont, en quelque sorte, le réveil de pratiques oubliées. C'est là qu’elles prennent tout leur sens. Le substrat historique de l'art des jardins est particulièrement riche et diversifié pour permettre la réinvention et l'éclosion de l'invention. En est-il de même pour le paysage ?

Art du milieu, mais aussi de vie et d'imaginaire public jusqu'à etre l'art du beau jeu des sensations (Kant, 1993), le paysage n'est pas l'enfance de l'art. Il est ce que l'on pourrait appeler le beau sens.

Comme nous l'avons souligné précédemment, le paysage est une complexité dont le tréfonds n'est que trop fixité et réminis- 
cence visuelle. L'évếnement est propice et essentiel à son invention. Condition de notre modernité, cette quête se nourrit avant tout de l"étonnement. Ce dernier en est le déclencheur. Nous devons nous étonner de ce qui s'offre à notre vue. Le regard est l'événement trop souvent oublié. La frénésie photographique aura certainement contribué à le banaliser. Mais nous devons aussi accepter ce phénomène de l'instantanéité, de la reproduction, du reconditionnement et du cadrage de l'in visu. Le rejet volontaire $\mathrm{d}^{+}$une forme de pollution photographique et la lassitude du clic sont par contre des faits actuels qui nous rappellent le besoin de reconduire l'idée de l'expérience au lieu.

Le mouvement et la lenteur, la distanciation et la proximité sont les quelques acteurs binômes d'une condition paysagère qui a pour dessein, entre autres, de contrer l'hypertrophie d'un présent vide : l'ennui (Ronfard, 1998).

Philippe Poullaouec-Gonidec s'occupe activement du développenent des pratiques du paysage. $l$ a participé à la conception de plusieurs espaces publics montréalais. Il est directeur de la Chaire en paysage et environnement de la faculte de l'aménagement.

Un comité de lecture a lu et accepté ce texte

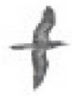

\section{NOTES}

1 A titre d'information, $\propto$ Le marche quebecois de fhorticulture ornementale corstitue wn sectear écononique important avec une consommation annuelle de plus de un milliard de dollars. L'industrie regroupe quelque 3500 entreprises de production et de commercialisation s (Le Journal Économique du Québec, septembre 1997).

2 D'après John Dixon Hunt (1996: 16), I'expression troisieme nature remonte à la Renaissance ( 1541 ) où elle fut utilisée pour conceptualiser le jardin d'agrément. Elle désigne a l...J Tintervention par laquelle, dans un espace donne, des hommes et des femmes façonnent et crétent un nowvel ewironnement pour eux-mentes ou pour une societe ou une culture donneses $x$

3 L'artial isation est un concept du philosophe Alan Roger (1978). Selon lui, il y a deux manières d'artialiser : l'artialisation in sizu qui consiste à inscrire le code artistique dans la matérialité du lieu et l'artialisation in visu qui est une opération indirecte sur le regard. auquel on fournit des modèles de vision, des schèmes de perception et de délectation.

4 Emmanuel Kant (1993), dans sa Critique de la faculte de juger, reprend une idée suggérée par Edmund Burke et transpose ces deux notions à saj définition de l'esthétique.

5 Térme emprunté par l"auteur à la définition du mot spectaculaire.

6 Voir, à ce sujet, les recherches réalisées pair la Chaire en paysage et environnement de I' Université de Montréal pour le ministère des Transports du Québec, soit celles concernant la Qualité paysagè re des corridors autoroutiers et routiers servant de portes d'entree importantes du Quebec (PERIQactivites / et 2 ), chercheurs $\mathrm{P}$. Jacobs, $\mathrm{M}$, Gariépy et P. Poullanoucc-Gonidec, et l' Étude de caracterisation et requalification des paysages d'enirée de la capirale du Québes (Corridor Duplessis), 1998, chercheurs P. Jacobs, P. Poullaouec-Gonidec et B. St-Denis.

7 Voir à titre d'exemple: Édouard, André (1879); et L. E. Audot (1859).

8 Lire à ce sujet Bernard Lassus (1977).

9 Citons à titre d'exemples les Floralies internationales a Montréal en 1980 et celles de la ville de Québec en 1996.

10 Bernard Lassus (France), Philippe Poullaouec-Gonidec (Canada), Anne Krol (Belgique) et Franco Zagari (Italie).

11 Cahier des charges des concepteurs (Commissariat de la Foire-Exposition de NiortFrance), 1992.

12 Cette manifestation est dirigée et organisée par le Conservatoire Intemational des Parcs et Jardins et du Paysage dont la mission déborde du festival puisqu'il développe des activités auprès du grand public (expositions. etc.), de la formation et de l'enseignement, des relations avec les professionnels, des activités de mise en valeut du patrimoine.

13 Le concours international, lancé chaque été. permet de recevoir 250 projets des cing continents. Prtésentés anonymement à un jury internationall, 15 à 20 projets sont sélectionnés et font l'objet d'une étude complémentaire de faisabilité, asvant que les choix definitif: soient fixés (source : communiqué de presse du Conservatoire, 1998).

14 Confirmation de la renaissance de l'art des jardins, la ville de Lausanne en Suisse rếalisait, cn 1997, une exposition publique de trente-quatre jardins dans les places, les rues et sur les talus de son métro. Ces jardins s'inscrivaient dans la ville (contrairement à Chaumont-sur-Loire, où ils sont éphémères) et étaient destinés à rester.

15 André Mollet (1981).

\section{BIBLIOGRAPHIE}

Audot, L.E. (1859), Traite de la composition el de l'ornement des jardins, Audot, librairieéditeur.

Baridon, M. (1998), Les jardins, Editions de Robert Laffont, Paris.

Burke, E. (1990), Recherche phitosophique sur l'origine de nos idees du sublinte et di beau. (Traduction Baldine de Saint-Girons), Paris, Vrin, réédition.

Clément, G. (1997), Thomas er le voyageur, Éditions Albin Michel.

Ẻdouard, A. (1879), Traité général de la composition des pares et des jardins, Edition Jeanne Laffitte, Marseille.

Hunt, J. D. (1996), L'art du jardin et son hiswore, Editions Odile Jacobs, 113 pages.

Kalaora, B. (1981), \& Le Musée vert *, Anthropos, réédition L'Harmattan, 1993.

Kalaora, B., et V. Pélosse (1977), \& La forết loisir, un équipement de pouvoir : l'exemple de la forêt de Fontainebleau $*$, Hérodote, 7 , juilletseptembre.

Kant, E. (1993), Critique de la faculte de juger (Traduction A. Philonenko), Paris, Vrin, réédition.

Laroze, C. (1990), Une histoire sensuelle des jardins, Editions Olivier Orban.

Lassus, B, (1977), Jardins imaginaires : les habitants paysagistes, Presse de la connaissance et Weber, Paris.

Lassus, B. (1994), Découvrir, s'arrêter, Autoroute et paysages, sous la direction de Bemard Leyrit et Bernard Lassus, Les Éditions du DemiCercle.

Mollet, A. (1981), Le jardin de plasiri, Edition du Moniteur.

Poullawec-Gonidec, P. et P. Jacobs (1992), "Le projet de paysage au Quebee $s, A R Q$. $A r$ chitecture Quebec, 63.

Poullaouec-Gonidec, P, (1993), * Esthétique des paysages de la modernité $\approx$. Trames, Revue de l'aménagement, dossier \& Le projet de paysage au Québec *, 9.

Roger, A. (1979), Nu ef paysages, essai sur la fonction de l'art, Édition Aubier, présence et pensée, 322 pages.

Ronfard, B. (1988), Eloge de l'étonnement, Éditions Desclée de Brouwer

Schelle, K. G. (1996), LArt de se promener. Éditions Rivages poche/Petite Bibliothèque. réédition.

Tournier, M. (1994), Le miroir des idees, Éditions Metcure de France. 